Michael Eduardo Reichenheim 1 Claudia Leite Moraes 1

\section{Adaptação transcultural do instrumento Parent-Child Conflict Tactics Scales (CTSPC) utilizado para identificar a violência contra a criança}

\author{
Portuguese-language cross-cultural \\ adaptation of the Parent-Child Conflict Tactics \\ Scales (CTSPC), an instrument used to identify \\ parental violence against children
}

1 Núcleo de Pesquisa das Violências, Departamento de Epidemiologia, Instituto de Medicina Social, Universidade do Estado do Rio de Janeiro. Rua São Francisco Xavier 524, 7o andar. Rio de Janeiro, RJ 20559-900, Brasil. michael@ims.uerj.br clmoraes@ims.uerj.br
Abstract This article concerns the first phase of the assessment of the cross-cultural equivalence between the Parent-Child Conflict Tactics Scales (CTSPC) designed in English and used to identify child abuse and neglect, and a Portuguese-language version to be used in Brazil. Evaluating conceptual and item equivalences involved expert groups. Assi sted by a broad literature re view, discussions focused on the existence and pertinence of the underlying theoretical concepts and the corresponding component items in the Brazilian context. The appraisal of semantic equival ence involved the following steps: two translations and respective back-translations; an evaluation regarding referential (literal) and general (meaning) equival ences between the original instrument and each version; further discussions with experts in order to produce a final version; and pre-testing the latter on $\mathbf{7 7 4}$ women. It proved possible to establish a satisfactory conceptual, item, and semantic equival ence between the Portuguese-language version and the original CTSPC. Acceptability of the version was excellent. Al beit encouraging, results should be reassessed in the light of forthcoming psychometric analysis (measurement equivalence), as well as through ongoing criticism from interested professionals.

Key words Domestic Violence; Child Abuse; Questionnaire

Resumo Este artigo enfoca a primeira fase da avaliação da equivalência transcultural entre o instrumento Parent-Child Conflict Tactics Scales, concebi do em ingl ês e usado para identificar violência contra a criança, e uma versão em português a ser proposta para uso no Brasil. Subsidiada por uma ampla revisão bi bliográfica, a avaliação da equival ência conceitual e de itens envolveu discussões com grupo de especialistas sobre a existência e pertinência em nosso meio dos concei tos teóri cos subj acentes e dos itens componentes do instrumento origi nal. A aval iação da equivalência semântica constou das seguintes etapas: duas traduções e respectivas re traduções; uma avaliação da equivalência de significado referencial (literal) e geral (sentido) entre as retraduções e o origi nal; novos en contros com especialistas para a defini ção de uma versão-síntese e um préteste realizado em 774 mulheres. Constatou-se boa equivalência conceitual de itens e semântica entre a versão final em português e o original, bem como uma excel ente aceitabilidade do instrumento adaptado. Apesar de encoraj adores, os resultados obtidos merecem ser revistos após aval iações psi cométricas futuras (equi valência de mensuração) e através de crítica contínua por parte dos profissionais interessados.

Palavras-chave Violência Doméstica; Maus-tratos Infantis; Questionário 


\section{Intro dução}

Em diferentes partes do mundo, a violência na família vem assumindo um crescente papel na morbi-mortalidade de mulheres, crianças e adolescentes (Heise, 1994; Moraes \& Reichenheim, 2002b; Rossman et al., 1999; Straus \& Gelles, 1995; Watts \& Zimmerman, 2002). Sua repercussão biopsicossocial tem fomentado um crescente interesse na elaboração de propostas de ação para a prevenção, acompanhamento de vítimas e no desenvolvimento de programas de investigação. Também no Brasil, há indicação de que a violência entre pais e filhos, um dos principais componentes da violência familiar, é um fenômeno expressivo, devendo ser encarada como prioridade na agenda dos diversos segmentos sociais (Deslandes et al., 2000; MS, 1997, 2001, 2002).

U $m$ dos principais desafios a enfrentar no campo da violência familiar é a identificação adequada dos eventos. Incentivos vêm sendo dados à elaboração de novos e mais acurados instrumentos de aferição, bem como ao aprimoramento dos já habitualmente utilizados no âmbito clínico e em contextos de pesquisa (Aldarondo \& Straus, 1994; Archer, 1999; Milner, 1994; Straus \& Hamby, 1997; Straus et al., 1998; Weiss et al., 2000). Aceitando-se que é de grande interesse comparar perfis epidemiológicos de diferentes culturas, vários autores recomendam que um instrumento consolidado em um certo contexto seja somente utilizado em outro após uma rigorosa adaptação que permita ligar e harmonizar os construtos e dimensões subjacentes (Behling \& Law, 2000; Guillemin et al., 1993; Herdman et al., 1998; Reichenheim \& Moraes, 2002).

As primeiras adaptações de instrumentos calcavam-se em simples traduções realizadas pelos próprios pesquisadores ou, quando muito, em processos de tradução-retradução nos quais se avaliava somente o grau de equivalência semântica entre o original e sua versão. Mais recentemente, diferentes estratégias vêm sendo propostas. A literatura sobre o tema tem aumentado progressivamente, principalmente às custas dos numerosos estudos comparativos internacionais desenvolvidos por pesquisadores das áreas de Antropologia e Sociologia (Behling \& Law, 2000). Dentre os diferentes métodos em debate, chama atenção o apresentado por Herdman et al. (1998) para adaptação de instrumentos na área de qualidade de vida e saúde. Por sua complexidade e discussão pormenorizada de diferentes aspectos da equivalência transcultural, este foi o modelo escolhido para a adaptação do instrumento focalizado no presente artigo.
Segundo os autores, o processo de adaptação deve iniciar com uma apreciação da pertinência dos conceitos e domínios apreendidos pelo instrumento original na cultura-alvo da nova versão (equivalência conceitual). O modelo também prescreve que se avalie a adequação de cada item proposto no instrumento original em termos da capacidade de representar tais conceitos e domínios na população-alvo (equivalência de itens). Somente então se passa à avaliação da equivalência semântica e lingüística entre os itens originais e vertidos (equivalência semântica). A adequação e a pertinência de aspectos operacionais - forma de administração, instruções, número de opções de resposta etc. - também necessitam de atenção (equivalência operacional). Na seqüência, avalia-se a equivalência entre as propriedades psicométricas do instrumento original e de sua nova versão (equivalência de mensuração).

Diante das excelentes avaliações anteriores das propriedades psicométricas das Conflict Tacti cs Scales (CTS) e de suas versões em diferentes idiomas (Archer, 1999; Assis, 1995; Hasselmann et al., 1998; Lucente et al., 2001; M oraes et al. , 2002; M oraes \& Reichenheim, 2002a; Straus, 1979; Straus et al., 1996), decidiu-se pela adaptação formal do instrumento Parent-Child Conflict Tactics Scales (CTSPC), específico para lidar com a relação entre pais e filhos. O instrumento foi concebido por Straus et al . (1998) em língua inglesa e consiste em um refinamento da primeira CTS, elaborada pelo Family Research Laboratory, nos Estados Unidos, há cerca de duas décadas (Straus, 1979). O módulo básico da CTSPC é composto por 22 itens divididos em três escalas - disciplina não-violenta, agressão psicológica e violência física. A última é subdividida em três, cada qual encampando atos de distintas gravidades - menores ou punição corporal; graves ou maus-tratos físicos e muito graves ou maus-tratos físicos grave. O instrumento compreende ainda três módulos suplementares que abordam as táticas utilizadas para disciplinamento infantil na semana anterior à entrevista, situações de negligência e abuso sexual.

Neste artigo, são enfocadas as três primeiras fases do processo de adaptação sugerido por Herdman et al. (1998), envolvendo somente o módulo básico do instrumento. Essa restrição se justifica, de um lado, pela magnitude da violência psicológica e física contra a criança em nosso meio e, de outro, pela insuficiência de informações sobre a acurácia das escalas complementares do instrumento original (negligência e abuso sexual). Também é um objetivo do artigo propor uma versão-síntese para testes e subseqüente uso no Brasil. 


\section{Métodos}

Avaliação da equivalência

conceitual e de itens

Essas duas fases de avaliação foram desenvolvidas de forma concomitante por um grupo de especialistas composto por cinco profissionais que atuam na área de violência familiar. O grupo procurou explorar se as diferentes dimensões abarcadas pelo instrumento original na definição e apreensão da violência contra a criança seriam relevantes e pertinentes em nosso meio. No processo, avaliou a pertinência dos itens do instrumento original para a captação de cada uma dessas dimensões da violência familiar no Brasil.

A apreciação focalizou especialmente o contexto brasileiro, abarcando os seguintes pontos: (1) a pertinência das táticas de disciplina não violenta, agressão psicológica e violência física na educação infantil; (2) a existência de outras estratégias de disciplinamento que, por serem relevantes no Brasil, devessem, necessariamente, ser incluídas em um instrumento epidemiológico de identificação da violência entre pais e filhos; (3) a adequação das duas dimensões presentes no questionário original (agressão psicológica e violência física) para expressar o conceito de violência e a necessidade de inclusão de outras; (4) a capacidade dos itens que compõem as três escalas de representar as dimensões abarcadas pelo instrumento e (5) a pertinência da classificação dos mesmos segundo a sua gravidade.

As discussões foram subsidiadas por uma revisão bibliográfica que priorizou as publicações das CTSs sobre os processos envolvidos em sua construção (Straus, 1979; Straus et al., 1996, 1998). Essa etapa visou conhecer os conceitos e definições teóricas de violência familiar em geral e, especificamente, contra a criança, assim como as estratégias utilizadas para a escolha dos itens componentes. Adicionalmente, procurou-se identificar as mesmas questões no material bibliográfico publicado pelas principais instituições nacionais envolvidas com a prevenção, diagnóstico e acompanhamento de vítimas de violência familiar (ABRAPIA, 1992; BEM FAM , 1998; Branco et al., 1996; Deslandes, 1997; MS, 2002).

Avaliação da equivalência semântica

Seguindo o mesmo roteiro descrito detalhadamente em estudo conexo (Moraes et al., 2002), a avaliação de equivalência semântica envolveu cinco etapas. A primeira consistiu na reali- zação de duas traduções do original em inglês para o português, executadas independentemente por profissionais bilíngües. $\mathrm{Na}$ segunda etapa do processo, as versões preliminares foram retraduzidas por outros dois profissionais, novamente de forma independente.

U ma avaliação externa foi efetuada por um quinto tradutor, não só proficiente e fluente nos dois idiomas, como também ligado à área de Saúde Coletiva (etapa 3). Duas facetas distintas, mas complementares, foram apreciadas: a equivalência entre o original e as retraduções quanto ao significado referencial (literal) dos termos constituintes e, para cada item, o significado geral (sentido) dos textos (Herdman et al., 1998). Em relação à primeira faceta, julgou-se a equivalência entre pares de assertivas de forma contínua ( $0 \%$ a $100 \%$ ) através de escalas visuais - Visual Analogue Scale (Streiner \& Norman, 1995). O significado geral foi qualificado em quatro níveis, a saber, inalterado, pouco alterado, muito alterado ou completamente alterado.

A quarta etapa da avaliação semântica foi realizada pelo mesmo grupo de especialistas que participou da avaliação da equivalência conceitual e de itens, tendo como objetivo identificar e solucionar os problemas surgidos nas etapas pregressas. A partir daí, pôde-se propor uma versão-síntese, ora incorporando itens oriundos de uma das duas versões trabalhadas, ora optando-se por introduzir novas modificações para melhor atender os critérios expostos acima.

A quinta e última etapa de avaliação envolveu o pré-teste da versão-síntese proposta. A CTSPC foi aplicada em estudo realizado em três maternidades públicas do Município do Rio de Janeiro (Moraes, 2001). Uma equipe composta por cinco entrevistadoras especialmente treinadas e pela coordenadora do trabalho de campo (CLM) entrevistou 774 mães nas primeiras 48 horas de puerpério, focalizando situações vivenciadas com outras crianças de convívio íntimo. Durante a coleta de dados, realizaram-se reuniões da equipe com o grupo de especialistas a fim de discutir a aceitabilidade da versão e demais observações decorrentes da aplicação do instrumento.

\section{Resultados e discussão}

As discussões do grupo de especialistas e a consulta bibliográfica indicaram que os conceitos relacionados à violência entre pais e fiIhos utilizados para a elaboração do instrumento original são pertinentes ao contexto 
cultural brasileiro. Concordando com o proposto por Straus et al. (1998), julgou-se que as definições e dimensões da violência contra a criança no Brasil também englobam, dentre outros aspectos, a agressão psicológica e a violência física.

Todavia, nas várias fases de atuação do grupo de especialistas, contemplou-se a inclusão de outras facetas da violência contra crianças e adolescentes na versão a ser usada no Brasil. Ficou a indagação se a prostituição ou o trabalho infantil não seriam também formas de maus-tratos e merecedoras de enfoque. Apesar de acordado que estas dimensões fazem parte de um quadro maior de violências, julgou-se que não seria interessante introduzi-las na versão, pois as mesmas não se restringem ao contexto dos conflitos de foro íntimo, foco central do instrumento original.

Segundo os especialistas, de forma geral, os 22 itens escolhidos para compor a CTSPC original também representam adequadamente as dimensões da violência contra a criança na população-alvo. Não obstante, o grupo debateu a possibilidade de inclusão de novos itens, que abarcassem outras ações de violência particularmente comuns em nosso meio. Ao se avaliar a escala de agressão psicológica, por exemplo, sentiu-se falta de perguntas que abordassem estratégias de amedrontamento. Pais freqüentemente lançam mão de figuras como "bichopapão" ou "homem do saco" para intimidar e modificar comportamentos julgados inadequados. Foram lembradas, também, certas estratégias atemorizantes e a utilização de figuras simbolicamente restritivas/ punitivas como forma de disciplinamento. "Trancar no quarto escuro" ou "chamar o guarda ou a polícia" são intimi dações freqüentes que talvez merecessem itens específicos. Ainda assim, a despeito dessas sugestões interessantes, entendeu-se que não seria prudente modificar a estrutura vigente do instrumento. Vale lembrar que a inclusão de um ou mais itens configura um novo instrumento, cujas propriedades psicométricas não são necessariamente equivalentes às do original.

Mesmo julgando todos os itens componentes da CTSPC pertinentes ao contexto cultural brasileiro, alguns mereceram um debate adicional, conforme apresentado na Tabela 1.0 item $L$, pertencente à escala agressão psicológica, por exemplo, não parece de todo adequado ao trato com crianças muito pequenas, pois a ameaça de expulsão de casa não cabe nessa situação. De forma semelhante, os itens D e H, da escala de castigo corporal, ainda que sejam táticas de disciplinamento bastante utilizadas em crianças pré-escolares e escolares, não costumam fazer parte das relações entre pais e fiIhos adolescentes.

A preocupação com a capacidade de generalização motivou a manutenção dos dois itens cogitados como pouco ajustados às diferentes idades-alvo do instrumento. Possivelmente, seria vantajoso haver módulos separados para menores de 12 anos e adolescentes, já que as estratégias utilizadas no disciplinamento variam conforme a idade. No entanto, além de acarretar um aumento do questionário, isso poderia prejudicar as comparações de achados obtidos no Brasil e em localidades que usam o instrumento na sua forma original. As discussões trazidas à baila no artigo que apresenta o instrumento original reforçaram essa decisão (Straus et al., 1998). Mesmo reconhecendo que a heterogeneidade das formas de violência psicológica e física nas diferentes faixas etárias tenha levado a alguma dificuldade na sua construção, os autores ressaltam que a diversidade dos itens componentes acaba permitindo uma representação adequada do espaço de conteúdo desejado nas diferentes faixas etárias. Enfatizam que as vantagens de um instrumento único e de ampla cobertura sobrepujam quaisquer limitações.

Questionou-se também a pertinência dos itens B e Q na representação do conceito de disciplina não-violenta em populações com precárias condições de vida, como é o caso de uma importante parcela da população brasileira. Famílias desse estrato social freqüentemente vivem em casas de poucos cômodos, em que crianças e adultos dividem espaços diminutos. Pôs-se em dúvida se, nessas circunstâncias, um item como o $B$, referindo-se designadamente ao quarto próprio da criança, não tenderia a diminuir a sensibilidade da detecção dessa estratégia de disciplinamento. Do mesmo modo, o termo "privilégios" contido no item Q também pode ser impróprio, já que a maioria das crianças menos favorecidas socialmente não tem sequer o essencial, quanto mais, supostas regalias. Decidiu-se fazer uma alteração semântica apenas no item $B$, acrescentando-Ihe "ou em qualquer outro lugar" para contemplar as famílias que, na perspectiva de infligir "castigo", utilizam outros ambientes domiciliares como local de restrição da criança. Como a crítica ao item Q não foi inteiramente compartiIhada por todo o grupo de especialistas, optouse por não modificá-lo.

Ainda nesta fase, debateu-se a alocação do item $V$ na escala de punição corporal. Segundo o grupo de especialistas, atos como dar uma bofetada na cara ou tapa na orelha parecem re- 
Problemas identificados durante o processo de avaliação da equivalência de itens e algumas modificações implementadas.

\begin{tabular}{|c|c|c|c|}
\hline Item original & Comentário & Tipo de problema & Ação \\
\hline $\begin{array}{l}\text { Put him in time out } \\
\text { (or sent to his room) (B) }\end{array}$ & $\begin{array}{l}\text { A maioria das famílias entrevistadas } \\
\text { mora em casas pequenas onde as } \\
\text { crianças não têm quarto próprio }\end{array}$ & $\begin{array}{l}\text { Prejuízo na equivalência } \\
\text { de item }\end{array}$ & $\begin{array}{l}\text { Modificação semântica: } \\
\text { "colocou-o de castigo do tipo: } \\
\text { mandou-o ficar em seu quarto } \\
\text { ou em qualquer outro lugar" }\end{array}$ \\
\hline $\begin{array}{l}\text { Hit him on the bottom with } \\
\text { something like a belt, } \\
\text { hairbrush, a stick or some } \\
\text { other hard object (D) }\end{array}$ & $\begin{array}{l}\text { O item não parece adequado } \\
\text { a crianças maiores }\end{array}$ & Problema no item original & Não modificado \\
\hline $\begin{array}{l}\text { Spanked him on the bottom } \\
\text { with your bare hand }(\mathrm{H})\end{array}$ & $\begin{array}{l}\text { O item não parece adequado } \\
\text { a crianças maiores }\end{array}$ & Problema no item original & $\mathrm{N}$ ão modificado \\
\hline Swore or cursed at him (J) & $\begin{array}{l}\text { No Brasil, o primeiro verbo não é } \\
\text { muito utilizado. O sentido do item } \\
\text { parece conotar violência mais } \\
\text { grave aqui do que nos Estados Unidos }\end{array}$ & $\begin{array}{l}\text { Prejuízo na equivalência } \\
\text { de item e semântica }\end{array}$ & $\begin{array}{l}\text { Modificação semântica: “xingou } \\
\text { ou praguejou, quer dizer, 'rogou } \\
\text { praga' contra ele" }\end{array}$ \\
\hline $\begin{array}{l}\text { Said you would send him } \\
\text { away or kick him } \\
\text { out of the house (L) }\end{array}$ & $\begin{array}{l}\mathrm{N} \text { ão parece adequado } \\
\text { às crianças pequenas }\end{array}$ & Problema no item original & Não modificado \\
\hline $\begin{array}{l}\text { Took away privileges } \\
\text { or grounded him (Q) }\end{array}$ & $\begin{array}{l}\text { No Brasil, o termo "privilégio" pode } \\
\text { significar algo sobressalente, supérfluo. } \\
\text { Caso o instrumento seja utilizado } \\
\text { em populações de baixa renda, } \\
\text { o item pode não conseguir captar } \\
\text { o mesmo conteúdo do original }\end{array}$ & $\begin{array}{l}\text { Prejuízo na equivalência } \\
\text { de item }\end{array}$ & Não modificado \\
\hline $\begin{array}{l}\text { Called him dumb } \\
\text { or lazy or some other } \\
\text { name like that }(U)\end{array}$ & $\begin{array}{l}\mathrm{N} \text { ão parece cobrir o mesmo conteúdo. } \\
\text { Talvez, no Brasil, outros adjetivos } \\
\text { depreciativos fossem mais indicados }\end{array}$ & $\begin{array}{l}\text { Prejuízo na equivalência } \\
\text { de item }\end{array}$ & $\begin{array}{l}\text { Modificação semântica: "chamou-o } \\
\text { de estúpido, burro, preguiçoso } \\
\text { ou de outra coisa parecida" }\end{array}$ \\
\hline $\begin{array}{l}\text { Slapped him on the } \\
\text { face, head or ears }(V)\end{array}$ & $\begin{array}{l}\mathrm{N} \text { ão parece pertencer ao construto } \\
\text { de violência física menor, mas, } \\
\text { sim, ao de violência física grave }\end{array}$ & $\begin{array}{l}\text { Prejuízo na equivalência } \\
\text { de item }\end{array}$ & $\mathrm{N}$ ão modificado \\
\hline
\end{tabular}

presentar uma situação de violência mais grave em virtude de suas possíveis conseqüências à saúde, principalmente quando utilizados contra crianças mais novas. O entendimento do que vem a ser castigo violento em contraposição ao que pode ser descrito como método coerente e aceitável para educação e disciplinamento de uma criança é um campo dinâmico, aberto e em franca discussão. Essas definições tendem a variar segundo o contexto social. Falta consenso sobre o que é ou não aceitável, e quais valores pessoais, culturais e religiosos que as influenciam (Bauman \& Friedman, 1998). Atos violentos inicialmente considerados graves vão sendo progressivamente trivializados, passando a ser aceitos e percebidos como necessários à "educação" de crianças.

É possível que este percurso tenha culminado com inclusão do item $\mathrm{V}$ na escala de vio- lência física menor. No contexto brasileiro, no entanto, há de se questionar se, pelo menos hoje, "dar um tapa ou bofetada no rosto, na cabeça ou nas orel has" pode ser considerado um ato corriqueiro. Talvez a melhor solução fosse transferir o item para a escala de maus-tratos físicos. No entanto, vale notar que, fazendo-se a substituição, a escala de violência física menor se reduziria, ao passo que a grave acabaria com um item a mais. Essa mudança poderia distorcer as propriedades psicométricas de ambas subescalas, um problema já assinalado anteriormente. Como no caso do item Q, optou-se por manter a estrutura original e aguardar os resultados das avaliações psicométricas para uma proposta mais definitiva.

Passando para a avaliação dos aspectos estritamente semânticos, concluiu-se que, em ambos os pares de tradução/ retradução, havia 
se alcançado uma adequada equivalência de significado referencial e geral vis-à-vis o instrumento original (etapa 3). Conforme mostra a Tabela 2, a maioria dos itens foi julgada como tendo mais de $90 \%$ de equivalência, com uma ligeira vantagem do primeiro sobre o segundo.

Apesar dessa avaliação positiva, 12 itens ainda demandaram pequenos ajustes durante a etapa subseqüente (etapa 4). Esse refinamento teve como objetivo tornar a versão mais sintonizada com a população-alvo e procurou evitar uma possível perda do sentido geral dos itens. A maioria das mudanças envolveu a substituição de pronomes e artigos (cerca de 70\%) para tornar o texto mais coloquial. Como exposto na Tabela 1, apenas três itens necessitaram ser alterados de forma mais substantiva. Além da mudança no item $B$, já comentada anteriormente, ao $U$ adicionou-se "estúpido" para ressaltar a idéia de que os adjetivos depreciativos propostos seriam apenas exemplos de um conjunto variado de termos utilizados com intuito de humilhar ou diminuir a auto-estima da criança. Ao J, acrescentou-se “...quer dizer, rogou praga..." no intuito de facilitar o entendimento.

Se as mudanças nos itens B e U parecem ter sido bem sucedidas, já que as respondentes não demonstraram dificuldade na compreensão dos itens, o mesmo não parece ter acontecido com o J. Apesar da modificação proposta pelo grupo de especialistas, o pré-teste continuou indicando um problema. As entrevistadoras, de forma consistente, relataram o quanto o item acarretava uma reação de espanto por parte das respondentes, dando-Ihes a impressão de que este parecia estar conotando uma situação mais grave do que a pretendida pelo instrumento original. Essa questão ainda carece de al guma luz. Seria importante realizar discussões que também incorporassem membros da população para a qual a versão foi pensada. Nesse sentido, grupos focais poderiam ser bastante proveitosos (Krueger, 1994; Chor et al., 2001).

Ainda no pré-teste, outros dois itens foram identificados como problemáticos. Segundo depoimento das entrevistadoras de campo, o item $A$, pertencente à escala de disciplina nãoviolenta, e o E, da escala de agressão psicológica, permaneciam pouco coloquiais. Unanimemente, a equipe apontou para a forma rebuscada de construção das duas sentenças e relatou que, em várias entrevistas, fora necessário repetir mais de uma vez essas perguntas para que se obtivesse resposta. Não obstante, observou-se uma excelente aceitabilidade do instrumento. Apenas duas entre as 774 respondentes se recusaram a terminar a entrevista. A versão pré-testada pode ser apreciada na íntegra na Tabela 3.

\section{Considerações finais}

As diferenças existentes entre as definições, crenças e comportamentos relacionados aos temas de pesquisa impõem que a utilização de instrumentos elaborados em outros contextos culturais seja precedida de uma avaliação meticulosa da equivalência entre o original e sua versão. A necessidade de se adaptarem ferramentas de aferição não é restrita às situações envolvendo países e/ou idiomas distintos. Ajustes locais e regionais também requerem atenção. O alcance de sintonia com a população na qual a versão será usada é algo difícil de caracterizar. Uma decisão deve ponderar o quanto se ganha com a aproximação cultural e o quanto se perde em termos de generalização e comparabilidade.

Como apontado na Introdução, historicamente as adaptações de instrumentos elaborados em outros idiomas se confinavam a simples traduções ou, excepcionalmente, à comparação literal dos originais com versões retraduzidas. Mais recentemente, pesquisadores de diferentes áreas temáticas têm sugerido que a avaliação semântica seja considerada apenas um dos passos necessários ao processo de adaptação transcultural (Badia \& Alonso, 1995; Behling \& Law, 2000; Bravo et al., 1991; Guillemin et al., 1993; Herdman et al., 1997). Ainda que na literatura se encontrem várias propostas metodológicas, utilizou-se o modelo sugerido por Herdman (1998) pela sua ênfase na apreciação de diferentes aspectos do processo de adaptação. Contudo, por ser apenas um dentre os vários roteiros disponíveis, torna-se fundamental que todas as fases/ etapas realizadas sejam explicitadas e postas à crítica.

Apreciando os diferentes passos do processo de avaliação da equivalência semântica, merecem destaques as duas últimas etapas. 0 envolvimento do grupo de especialistas em uma nova rodada de encontros serviu como um momento crucial de reflexões e debates, contribuindo para um ajuste fino. A importância desta quarta etapa é facilmente identificada ao se perceber que 12 entre os 22 itens sofreram alguma mudança no decorrer do processo, a despeito de uma avaliação satisfatória na anterior. É possível que as semelhanças de estrutura lingüísticas entre os idiomas, ou mesmo a presença de um jargão, tenham facilitado o processo de retradução, aproximando ao original sem 
Equivalência semântica entre a versão em português da Parent-Conflict Tactics Scales (CTSPC) e o original em inglês.

\begin{tabular}{|c|c|c|c|c|c|c|c|c|}
\hline \multirow{2}{*}{$\begin{array}{l}\text { Formulário original } \\
\text { (inglês) }\end{array}$} & \multicolumn{4}{|c|}{ Versão 1} & \multicolumn{4}{|c|}{ Versão 2} \\
\hline & $\begin{array}{l}\text { Tradução } \\
\text { (inglês } \rightarrow \text { português) }\end{array}$ & $\begin{array}{l}\text { Retradução } \\
\text { (português } \rightarrow \text { inglês) }\end{array}$ & $A 1^{a}$ & $\mathrm{~A} 2 \mathrm{~b}$ & $\begin{array}{l}\text { Tradução } \\
\text { (inglês } \rightarrow \text { português) }\end{array}$ & $\begin{array}{l}\text { Retradução } \\
\text { (português } \rightarrow \text { inglês) }\end{array}$ & Ala & $\mathrm{A} 2 \mathrm{~b}$ \\
\hline $\begin{array}{l}\text { a) Explained why } \\
\text { something was wrong }\end{array}$ & $\begin{array}{l}\text { Explicou por que } \\
\text { alguma coisa } \\
\text { estava errada }\end{array}$ & $\begin{array}{l}\text { You explained to } \\
\text { him why something } \\
\text { was wrong }\end{array}$ & 100 & IN & $\begin{array}{l}\text { Explicou por que } \\
\text { algo estava errado }\end{array}$ & $\begin{array}{l}\text { You explained why } \\
\text { something was wrong }\end{array}$ & 100 & IN \\
\hline $\begin{array}{l}\text { b) Put him in "time out" } \\
\text { (or sent to his room) }\end{array}$ & $\begin{array}{l}\text { Colocou-o "de molho" } \\
\text { (ou mandou-o para } \\
\text { o seu quarto) }\end{array}$ & $\begin{array}{l}\text { You grounded him (or } \\
\text { sent him to his room) }\end{array}$ & 100 & IN & $\begin{array}{l}\text { Colocou-o de castigo } \\
\text { (ou mandou-o } \\
\text { ficar no quarto) }\end{array}$ & $\begin{array}{l}\text { You punished } \\
\text { him or made him } \\
\text { stay in his room }\end{array}$ & 85 & IN \\
\hline c) Shook him & Sacudiu-o & You shook him hard & 95 & IN & Sacudiu-o & You shook him & 100 & IN \\
\hline $\begin{array}{l}\text { d) Hit him on the bottom } \\
\text { with something like } \\
\text { a belt, hairbrush, } \\
\text { a stick or some other } \\
\text { hard object }\end{array}$ & $\begin{array}{l}\text { Bateu no traseiro } \\
\text { dele/a com alguma } \\
\text { coisa como um cinto, } \\
\text { escova de cabelo, } \\
\text { vara ou outro objeto } \\
\text { duro }\end{array}$ & $\begin{array}{l}\text { You hit him on his } \\
\text { backside (spanked him) } \\
\text { with something like } \\
\text { a belt, a hairb rush, } \\
\text { a rod or any other } \\
\text { hard object }\end{array}$ & 100 & IN & $\begin{array}{l}\text { Bateu nele por trás, } \\
\text { com um cinto, uma } \\
\text { escova, um bastão } \\
\text { ou outro objeto duro }\end{array}$ & $\begin{array}{l}\text { You hit him on the } \\
\text { back, with a belt, } \\
\text { brush, bat or any } \\
\text { other hard object }\end{array}$ & 50 & PA \\
\hline $\begin{array}{l}\text { e) Gave him something } \\
\text { else to do instead of } \\
\text { what he/she was doing } \\
\text { wrong }\end{array}$ & $\begin{array}{l}\text { Deu-lhe outra coisa } \\
\text { para fazer em vez } \\
\text { daquilo que estava } \\
\text { fazendo errado }\end{array}$ & $\begin{array}{l}\text { You gave him } \\
\text { something else to do } \\
\text { instead of whatever he } \\
\text { was doing that was } \\
\text { wrong }\end{array}$ & 100 & IN & $\begin{array}{l}\text { Mandou-o fazer algo } \\
\text { diferente do que ele } \\
\text { estava fazendo errado }\end{array}$ & $\begin{array}{l}\text { He was doing } \\
\text { something wrong, } \\
\text { and you told him to } \\
\text { do something else }\end{array}$ & 100 & IN \\
\hline $\begin{array}{l}\text { f) Shouted, yelled, } \\
\text { or screamed at him }\end{array}$ & $\begin{array}{l}\text { Gritou, berrou, urrou } \\
\text { com ele }\end{array}$ & $\begin{array}{l}\text { You shouted, yelled, } \\
\text { or hollered at him }\end{array}$ & 100 & IN & $\begin{array}{l}\text { Falou alto, berrou } \\
\text { ou gritou com ele }\end{array}$ & $\begin{array}{l}\text { You spoke loud, } \\
\text { screamed or } \\
\text { yelled at him }\end{array}$ & 100 & IN \\
\hline $\begin{array}{l}\text { g) Hit him with a fist or } \\
\text { kicked him hard }\end{array}$ & $\begin{array}{l}\text { Acertou-o com o punho } \\
\text { ou deu-lhe um chute } \\
\text { com força }\end{array}$ & $\begin{array}{l}\text { You struck him with } \\
\text { your fist, or kicked } \\
\text { him hard }\end{array}$ & 100 & IN & $\begin{array}{l}\text { Bateu nele com a } \\
\text { mão fechada ou } \\
\text { bateu nele com força }\end{array}$ & $\begin{array}{l}\text { You hit him hard } \\
\text { with a closed hand }\end{array}$ & 90 & IN \\
\hline $\begin{array}{l}\text { h) Spanked him on the } \\
\text { bottom with your bare } \\
\text { hand }\end{array}$ & $\begin{array}{l}\text { Deu um tapa com } \\
\text { a palma da mão } \\
\text { no seu traseiro }\end{array}$ & $\begin{array}{l}\text { You slapped him on } \\
\text { the backside with the } \\
\text { palm of your hand }\end{array}$ & 100 & IN & $\begin{array}{l}\text { Bateu nas nádegas } \\
\text { dele com as mãos }\end{array}$ & $\begin{array}{l}\text { You hit his buttocks } \\
\text { with both hands }\end{array}$ & 96 & IN \\
\hline $\begin{array}{l}\text { i) Grabbed him around } \\
\text { the neck and choked him }\end{array}$ & $\begin{array}{l}\text { Agarrou-o pelo pescoço } \\
\text { e o sufocou }\end{array}$ & $\begin{array}{l}\text { You grabbed him by } \\
\text { the neck and } \\
\text { smothered him }\end{array}$ & 100 & IN & $\begin{array}{l}\text { Agarrou-o pelo } \\
\text { pescoço e sacudiu }\end{array}$ & $\begin{array}{l}\text { You grabbed him by } \\
\text { the neck and shook him }\end{array}$ & 79 & IN \\
\hline $\begin{array}{l}\text { j) Swore or cursed } \\
\text { at him }\end{array}$ & $\begin{array}{l}\text { Xingou-o ou } \\
\text { amaldiçoou-o }\end{array}$ & $\begin{array}{l}\text { You abused him } \\
\text { or cursed him }\end{array}$ & 94 & PA & $\begin{array}{l}\text { Xingou ou praguejou } \\
\text { contra ele }\end{array}$ & $\begin{array}{l}\text { You insulted him } \\
\text { or swore }\end{array}$ & 100 & IN \\
\hline $\begin{array}{l}\text { k) Beat him up, that is } \\
\text { you hit him over and } \\
\text { over as hard as you } \\
\text { could }\end{array}$ & $\begin{array}{l}\text { Deu-Ihe uma surra, ou } \\
\text { seja, bateu nele "sem } \\
\text { parar" com o máximo } \\
\text { de força que podia }\end{array}$ & $\begin{array}{l}\text { You gave him a severe } \\
\text { beating, that is, you hit } \\
\text { him relentlessly and as } \\
\text { hard as you could }\end{array}$ & 100 & IN & $\begin{array}{l}\text { Bateu muito nele, } \\
\text { isto é, bateu nele s } \\
\text { em parar, o máximo } \\
\text { que conseguiu }\end{array}$ & $\begin{array}{l}\text { You hit him hard } \\
\text { and repeatedly, as } \\
\text { much as you could }\end{array}$ & 100 & IN \\
\hline $\begin{array}{l}\text { I) Said you would send } \\
\text { him away or kick him } \\
\text { out of the house }\end{array}$ & $\begin{array}{l}\text { Disse que iria mandá-lo } \\
\text { embora ou para fora } \\
\text { de casa }\end{array}$ & $\begin{array}{l}\text { You said you would } \\
\text { send him away or throw } \\
\text { him out of the house }\end{array}$ & 100 & IN & $\begin{array}{l}\text { Disse que iria expulsá-lo } \\
\text { de casa ou enxotá-lo } \\
\text { para fora de casa }\end{array}$ & $\begin{array}{l}\text { You said you would } \\
\text { throw or kick him out } \\
\text { of the house }\end{array}$ & 100 & IN \\
\hline $\begin{array}{l}\text { m) Burned or scalded } \\
\text { him on purpose }\end{array}$ & $\begin{array}{l}\text { Queimou-o ou } \\
\text { escaldou-o } \\
\text { de propósito }\end{array}$ & $\begin{array}{l}\text { You burnt or scalded } \\
\text { him on purpose }\end{array}$ & 100 & IN & $\begin{array}{l}\text { Queimou-o ou } \\
\text { derramou líquido } \\
\text { quente nele de } \\
\text { propósito }\end{array}$ & $\begin{array}{l}\text { You burnt him or } \\
\text { spilled hot liquid } \\
\text { on him on purpose }\end{array}$ & 100 & IN \\
\hline $\begin{array}{l}\text { n) Threatened to spank } \\
\text { or hit him but did not } \\
\text { actually do it }\end{array}$ & $\begin{array}{l}\text { Ameaçou dar um } \\
\text { tapa mas não o } \\
\text { fez na verdade }\end{array}$ & $\begin{array}{l}\text { You threatened to slap } \\
\text { him but did not } \\
\text { actually do it }\end{array}$ & 100 & IN & $\begin{array}{l}\text { A meaçou espancá-lo } \\
\text { ou bater nele mas } \\
\text { não o fez }\end{array}$ & $\begin{array}{l}\text { You threatened to } \\
\text { spank or hit him } \\
\text { but did not do so }\end{array}$ & 100 & IN \\
\hline
\end{tabular}

(continua) 
Tabela 2 (continuação)

\begin{tabular}{|c|c|c|c|c|c|c|c|c|}
\hline \multirow{2}{*}{$\begin{array}{l}\text { Formulário original } \\
\text { (inglês) }\end{array}$} & \multicolumn{4}{|c|}{ Versão 1} & \multicolumn{4}{|c|}{ Versão 2} \\
\hline & $\begin{array}{l}\text { Tradução } \\
\text { (inglês } \rightarrow \text { português) }\end{array}$ & $\begin{array}{l}\text { Retradução } \\
\text { (português } \rightarrow \text { inglês) }\end{array}$ & Ala & $\mathrm{A} 2 \mathrm{~b}$ & $\begin{array}{l}\text { Tradução } \\
\text { (inglês } \rightarrow \text { português) }\end{array}$ & $\begin{array}{l}\text { Retradução } \\
\text { (português } \rightarrow \text { inglês) }\end{array}$ & Ala & $\mathrm{A} 2 \mathrm{~b}$ \\
\hline $\begin{array}{l}\text { o) Hit him on some other } \\
\text { part of the body besides } \\
\text { the bottom with } \\
\text { something like a belt, } \\
\text { hairbrush, a stick or } \\
\text { some other hard object }\end{array}$ & $\begin{array}{l}\text { Bateu-lhe em alguma } \\
\text { parte do corpo além } \\
\text { do traseiro, com } \\
\text { alguma coisa como } \\
\text { um cinto, escova de } \\
\text { cabelo, vara ou outro } \\
\text { objeto duro }\end{array}$ & $\begin{array}{l}\text { You whipped him } \\
\text { on some other part } \\
\text { of the body besides } \\
\text { his behind, with } \\
\text { something like a belt, } \\
\text { a hairbrush, a rod or } \\
\text { any other hard object }\end{array}$ & 100 & IN & $\begin{array}{l}\text { Bateu nele em outra } \\
\text { parte do corpo além } \\
\text { das nádegas, com } \\
\text { algo como cinto, } \\
\text { escova de cabelo, } \\
\text { bastão ou outro } \\
\text { objeto duro }\end{array}$ & $\begin{array}{l}\text { You hit some other } \\
\text { part of his body } \\
\text { besides his buttocks, } \\
\text { with a belt, hairbrush, } \\
\text { bat or any other } \\
\text { hard object }\end{array}$ & 82 & IN \\
\hline $\begin{array}{l}\text { p) Slapped him on the } \\
\text { hand, arm, or leg }\end{array}$ & $\begin{array}{l}\text { Deu-Ihe um tapa na } \\
\text { mão, braço ou perna }\end{array}$ & $\begin{array}{l}\text { You slapped him on } \\
\text { his hand, arm or leg }\end{array}$ & 100 & IN & $\begin{array}{l}\text { Deu um tapa na mão, } \\
\text { braço ou perna dele }\end{array}$ & $\begin{array}{l}\text { You slapped his } \\
\text { hand, arm or leg }\end{array}$ & 100 & IN \\
\hline $\begin{array}{l}\text { q) Took away privileges } \\
\text { or grounded him }\end{array}$ & $\begin{array}{l}\text { Tirou-lhe os privilégios } \\
\text { ou deixou-o sem sair }\end{array}$ & $\begin{array}{l}\text { You withdrew the } \\
\text { privileges he enjoyed } \\
\text { or forbade him to go out }\end{array}$ & 100 & IN & $\begin{array}{l}\text { Tirou privilégios } \\
\text { ou o reteve em casa }\end{array}$ & $\begin{array}{l}\text { You deprived him } \\
\text { of privileges or made } \\
\text { him stay home }\end{array}$ & 100 & IN \\
\hline r) Pinched him & Deu-Ihe um beliscão & You pinched him & 100 & IN & Deu-Ihe um beliscão & You pinched him (her) & 100 & IN \\
\hline $\begin{array}{l}\text { s) Threatened him } \\
\text { with a knife or gun }\end{array}$ & $\begin{array}{l}\text { Ameaçou-o com uma } \\
\text { faca ou arma de fogo }\end{array}$ & $\begin{array}{l}\text { You threatened him } \\
\text { with a knife or firearm }\end{array}$ & 100 & IN & $\begin{array}{l}\text { Ameaçou-o com } \\
\text { uma faca ou arma }\end{array}$ & $\begin{array}{l}\text { You threatened him } \\
\text { with a knife or a gun }\end{array}$ & 100 & IN \\
\hline $\begin{array}{l}\text { t) Threw or knocked } \\
\text { him down }\end{array}$ & Jogou-o no chão & $\begin{array}{l}\text { You threw him } \\
\text { on the floor }\end{array}$ & 100 & IN & $\begin{array}{l}\text { Atirou-o ao chão } \\
\text { ou bateu nele, } \\
\text { jogando-o ao chão }\end{array}$ & $\begin{array}{l}\text { You threw him on the } \\
\text { floor or hit him throwing } \\
\text { him on the floor }\end{array}$ & 100 & IN \\
\hline $\begin{array}{l}\text { u) Called him dumb } \\
\text { or lazy or some other } \\
\text { name tike that }\end{array}$ & $\begin{array}{l}\text { Chamou-o de estúpido } \\
\text { (burro) ou preguiçoso } \\
\text { ou de outra coisa } \\
\text { parecida }\end{array}$ & $\begin{array}{l}\text { You called him stupid } \\
\text { (dumb), or lazy, or } \\
\text { something of the kind }\end{array}$ & 100 & IN & $\begin{array}{l}\text { Chamou-o de pateta } \\
\text { ou preguiçoso ou } \\
\text { coisa parecida }\end{array}$ & $\begin{array}{l}\text { You called him stupid } \\
\text { or lazy or a similar } \\
\text { name }\end{array}$ & 100 & IN \\
\hline $\begin{array}{l}\text { v) Slapped him on the } \\
\text { face or head or ears }\end{array}$ & $\begin{array}{l}\text { Deu-lhe uma bofetada } \\
\text { na cara, cabeça ou } \\
\text { orelha }\end{array}$ & $\begin{array}{l}\text { You slapped him across } \\
\text { the face, head or over } \\
\text { the ear }\end{array}$ & 100 & IN & $\begin{array}{l}\text { Deu-lhe um tapa } \\
\text { no rosto, na cabeça } \\
\text { ou na sorelhas }\end{array}$ & $\begin{array}{l}\text { You slapped him on } \\
\text { his face, head or ears }\end{array}$ & 100 & IN \\
\hline
\end{tabular}

a A1 $\rightarrow$ Avaliação/apreciação em termos do percentual de concordância do significado referencial (ver texto para detalhes)

b A2 $\rightarrow$ Avaliação/apreciação do significado geral (ver texto para detalhes)

IN = inalterado; $P A=$ pouco alterado; $M A=$ muito alterado; $C A=$ completamente alterado.

que isso implicasse, necessariamente, uma versão bem adaptada ao contexto cultural local.

Foi também nesse momento que os problemas que levaram à falta de equivalência entre o original e as retraduções puderam ser identificados. Eles foram decorrentes de falhas na tradução, retradução ou em ambas as etapas. O exame cuidadoso impediu o inadvertido descarte de itens bem traduzidos, mas que, na realidade, foram mal retraduzidos. Algumas incongruências também puderam ser identificadas pelo grupo de especialistas. Por exemplo, apesar de os significados gerais dos itens C, G, $\mathrm{H}$ e O terem sido considerados "inalterados" pelo avaliador externo, identificou-se que as diferenças de significado referencial implicavam modificações reais no sentido dos itens. Talvez, o lapso na terceira etapa tenha se dado pela pouca experiência do profissional na área de violência familiar. Isso corroboraria Behling \& Law (2000), que, ao debaterem as várias fases de um processo de adaptação, propõem a incorporação dos tradutores ao programa de investigação. Enfatizam que, fazendo-os participar mais intensa e dinamicamente de todas as etapas do processo de adaptação, as experiências dos pesquisadores e as especificidades da área temática podem ser mais bem discutidas por todo o grupo, aprimorando o produto final.

A relevância da quinta fase, envolvendo as entrevistadoras de campo como partícipes do processo de adaptação, é nitidamente expressa no valioso feedback dado aos itens A, E e J. As informações carreadas sobre as reações verbais e não-verbais a cada pergunta; sobre as possíveis dissintonias entre os textos "neutros" e o conteúdo efetivamente percebido e sentido pelas respondentes; as críticas e sugestões semânticas ou operacionais apresentadas durante as muitas reuniões de trabalho agendadas ao longo do pré-teste, foram e continuarão sendo fundamentais na construção de um instru- 
Versão-síntese do intrumento Conflict Tactics Scales - Parent-Child (CTSPC) em português.

"Crianças muitas vezes fazem coisas que são erradas, desobedecem, ou fazem os pais ficarem zangados. A gente gostaria de saber o que V/S (ou $\mathrm{mmm}$ - pessoa escolhida/sorteada) costuma fazer quando o (xxx - nome da criança) faz alguma coisa errada, ou faz V/S ficar irritada ou zangada. Eu vou ler algumas coisas que V/S pode ter feito nessas horas. Eu gostaria de saber se, desde que a V/S soube que estava grávida, essas coisas aconteceram."

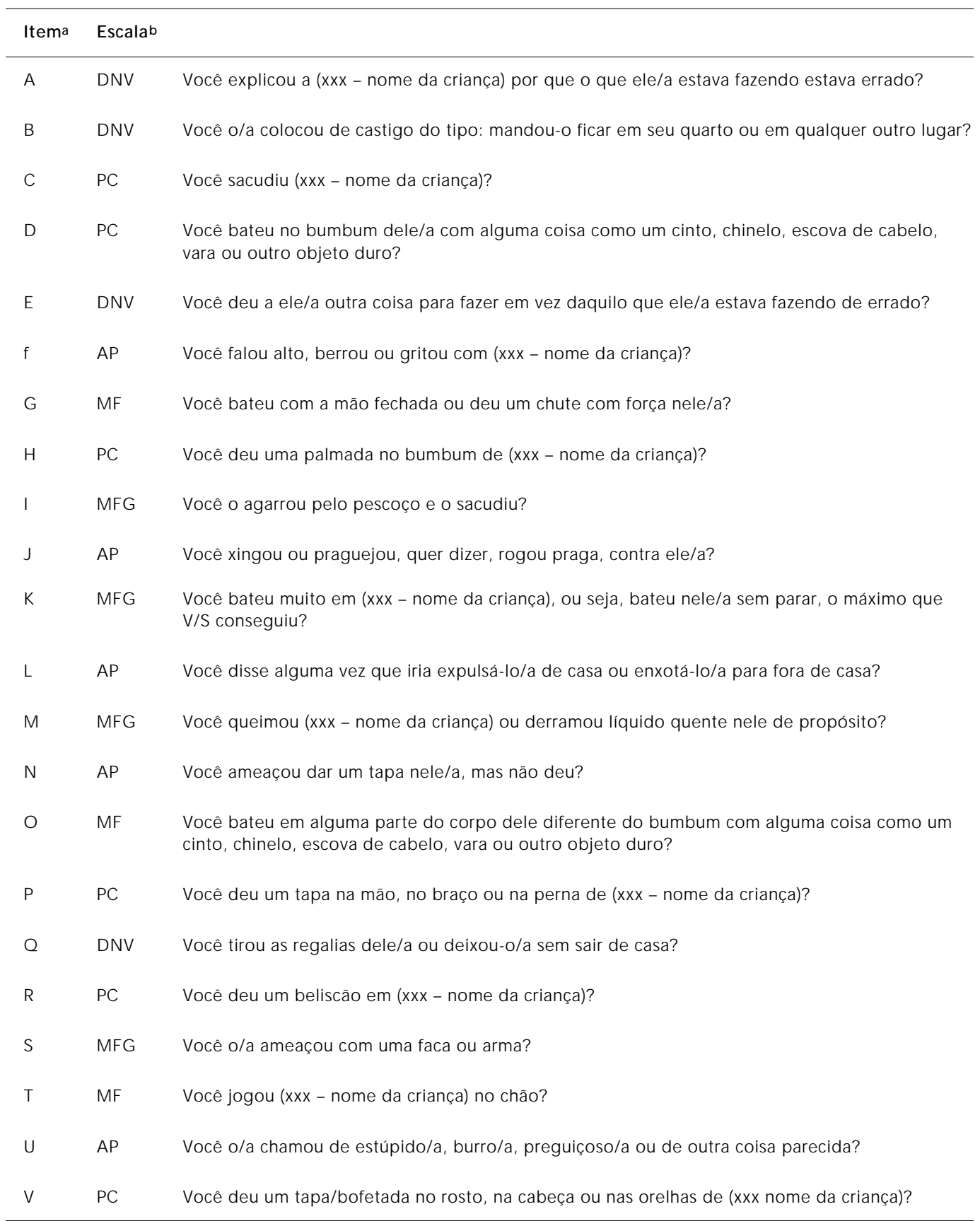

aLetras correspondem às do instrumento original em inglês (Straus et al., 1998). DNV = disciplina não-violenta; $A P=$ agressão psicológica; $P C=$ punição corporal; $\mathrm{MF}=$ maus-tratos físicos; $\mathrm{MFG}=$ maus-tratos físicos graves 
mento ainda mais conectado com a populaçãoalvo. De toda a sorte, essa experiência mostra que a proposta de Behling \& Law (2000), comentada acima, merece ser estendida também aos trabalhadores de campo. Em estudos futuros, seria interessante dar ainda mais um passo e integrá-los também às jornadas de debates aqui descritas na quarta etapa.

Divergências de apreciação nas diferentes etapas ratificam a importância da explicitação e crítica das estratégias de adaptação utilizadas. A falta de um roteiro único impõe que se debatam experiências, divulgando aquelas bem-sucedidas. Se dentro de um mesmo país já se observam importantes diferenças sócioculturais que podem diminuir a validade de instrumentos pouco adaptados ao contexto local, a utilização dos elaborados em outras culturas requer atenção redobrada. Torna-se cada vez mais evidente que uma simples tradução não é o suficiente. $O$ alerta é ainda mais pertinente em áreas temáticas culturalmente modeladas, como é o caso da violência familiar e tantas outras abordadas no campo da Saúde Coletiva.

Com base na presente avaliação da equivalência conceitual, de itens e semântica da CTSPC, é oportuno propor essa primeira versão em português. Contudo, ainda restam alguns passos importantes para que se possa aceitar com segurança a existência de equivalência transcultural entre o original e a versão. Como indicado na Introdução, a apreciação da equivalência de mensuração, estudada através da comparação entre as propriedades psicométricas da versão em português e do instrumento original, é uma tarefa essencial nesse processo e será implementada proximamente. Não obstante, é fundamental que essa primeira versão seja posta à crítica pelos profissionais interessados. A vasta gama de detalhes e opções, muitas delas intrinsecamente subjetivas, demanda que o aprimoramento do instrumento adaptado seja guiado por debates e negociações contínuas entre pares.

\section{Agradecimentos}

Este estudo foi parcialmente financiado pelo Consel ho Nacional de Desenvolvimento Científico e Tecnológico (CNPq - Programa de Apoio a Núcleos de Excelência), processo 663073/9987, e Fundação Carlos Chagas Filho de Amparo à Pesquisa do Estado do Rio de Janeiro, processos E-26/171.223/98 e E-26/ 150.893/ 99. M. E. R. foi parcialmente apoiado pelo CN Pq, processo 300234/94-5. C. L. M. teve o apoio parcial da Escola Nacional de Saúde Pública, Fundação Oswaldo Cruz. Os autores agradecem aos participantes do grupo de especialistas, Maria Helena Hasselmann, Simone de Assis e Barbara Musumeci Soares, pela disponibilidade em participar do estudo e pela riqueza de suas contribuições; a Christopher Peterson, por sua engajada participação na terceira etapa de avaliação da equivalência semântica, e às entrevistadoras Alessandra Veggi, Fernanda Faria de Almeida, Isabella Mota Rezende, Joelma Castro e Juliana Toshica Kunisawa, pelos valiosos comentários oferecidos durante a fase de pré-teste do instrumento. 


\section{Referências}

ABRAPIA (Associação Brasileira de Proteção à Infância e Adolescência), 1992. Maus-Tratos contra Crianças e Adol escentes. Proteção e Prevenção. Guia de Orientação para Profissionais de Saúde. Rio de Janeiro: ABRAPIA.

ALDARONDO, E. \& STRAUS, M. A., 1994. Screening for physical violence in couple therapy: Methodological, practical, and ethical considerations. Family Process, 33:425-439.

ARCHER, J., 1999. Assessment of the reliability of the conflict tactics scales: A meta-analytic review. Journal of Interpersonal Violence, 14:1263-1289.

ASSIS, S. G., 1995. Violência doméstica. Estudo epidemiológico sobre a vitimização física dos adolescentes de Duque de Caxias, Rio de Janeiro. Medicina, 28:51-60.

BADIA, X. \& ALONSO, J., 1995. Re-scaling the Spanish version of the sickness impact profile: An opportunity for the assessment of cross-cultural equivalence. Journal of Clinical Epidemiology, 48:949-957.

BAU MAN, L. J. \& FRIEDMAN, S. B., 1998. Corporal punishment. Pediatric Clinics of North America, 45:403-413.

BEHLING, O. \& LAW, K. S., 2000. Translating Questionnaires and other Research Instruments. v. 133, Thousand Oaks: Sage Publications.

BEMFAM (Sociedade Civil Bem-Estar Familiar no Brasil), 1998. Cidadão Adol escente. Rio de Janeiro: BEMFAM.

BRANCO, V. M. C.; PHEBO, L.; GOMES, M. A. S. M.; CASTRO, A. M. M. \& NOGUEIRA, M. A., 1996. Protegendo Nossas Crianças e Adolescentes. Rio de Janeiro: Secretaria Municipal de Saúde do Rio de Janeiro.

BRAVO, M.; CANINO, G. J.; RUBIO-STIPEC, M. \& WOODBURY-FARINA, M., 1991. A cross-cultural adaptation of a psychiatric epidemiologic instrument: The diagnostic interview schedule's adaptation in Puerto Rico. Culture, Medicine and Psychiatry, 15:1-18.

CHOR, D.; GRIEP, R. H.; LOPES, C. S. \& FAERSTEIN, E., 2001. Medidas de rede e apoio social no Estudo Pró-Saúde: pré-testes e estudo-piloto. Cadernos de Saúde Pública, 17:109-118.

DESLANDES, S. F., 1997. Prevenir a Violência: Um Desafio para Profissionais de Saúde. Rio de Janeiro: Centro Latino-Americano de Estudos sobre Violência e Saúde Jorge Careli, Escola nacional de Saúde Pública, Fundação Oswaldo Cruz.

DESLANDES, S. F.; GOMES, R. \& SILVA, C. M. F. P., 2000. Caracterização dos casos de violência doméstica contra a mulher atendidos em dois hospitais públicos do Rio de Janeiro. Cadernos de Saúde Pública, 16:129-137.

GUILLEMIN, F.; BOMBARDIER, C. \& BEATON, D., 1993. Cross-cultural adaptation of health-related quality of life measures: Literature review and proposed guidelines. Journal of Clinical Epidemiology, 46:1417-1432.

HASSELMANN, M. H.; LOPES, C. S. \& REICHENHEIM, M. E., 1998. Confiabilidade das aferições de um estudo sobre violência familiar e desnutrição severa na infância. Revista de Saúde Pública, 32:437-446.
HEISE, L., 1994. Gender-based abuse: The global epidemic. Cadernos de Saúde Pública, 10:135-145.

HERDM AN, M.; FOX-RUSHBY, J. \& BADIA, X., 1997. "Equivalence" and the translation and adaptation of health-related quality of life questionnaires. Quality of Life Research, 6:237-247.

HERDM AN, M.; FOX-RUSHBY, J. \& BADIA, X., 1998. A model of equivalence in the cultural adaptation of HRQoL instruments: The universalist approach. Quality of Life Research, 7:323-335.

KRUEGER, R., 1994. Focus Groups: A Practical Guide for Applied Research. 2a Ed. London: SAGE Publications.

LUCENTE, S. W.; FALS-STEWART, W.; RICHARDS, H. J. \& GOSCHA, J., 2001. Factor structure and reliability of the Revised Conflict Tactics Scales for incarcerated female substance abusers. Journal of Family Violence, 16:437-450.

MILNER, J. S., 1994. Assessing physical child abuse risk: The child abuse potential inventory. Clinical Psychology Review, 14:547-583.

MORAES, C. L., 2001. Aspectos M etodológicos Relacionados a um Estudo sobre Violência Familiar durante a Gestação como Fator de Propensão da Prematuridade no Recém-nascido. Tese de Doutorado, Rio de Janeiro: Escola Nacional de Saúde Pública, Fundação Oswaldo Cruz.

MORAES, C. L. \& REICHENHEIM, M. E., 2002a. Cross-cultural measurement equivalence of the Revised Conflict Tactics Scales (CTS2) Portuguese version used to identify violence within couples. Cadernos de Saúde Pública, 18:783-796.

MORAES, C. L. \& REICHENHEIM, M. E., 2002b. Domestic Violence During Pregnancy in Rio de Janeiro, Brazil. International Journal of Gynaecology and Obstetrics, 79:269-277.

MORAES, C. L.; HASSELM ANN, M. H. \& REICHENHEIM, M. E., 2002. Adaptação transcultural para o português do instrumento "Revised Conflict Tactics Scales (CTS2)", utilizado para identificar a violência entre casais. Cadernos de Saúde Pública, 18:163-176.

MS (Ministério da Saúde), 1997. Violência contra a Criança e o Adol escente. Proposta Preliminar de Prevenção e Assistência à Violência Doméstica. Brasília: MS.

MS (Ministério da Saúde), 2001. Política Nacional de Redução da Morbimortalidade por Acidentes e Violências. Brasília: MS.

MS (Ministério da Saúde), 2002. Notificação de MausTratos contra Crianças e Adol escentes pel os Profissionais de Saúde: U m Passo a mais na Cidadania em Saúde. v. 167, Brasília: Secretaria de Assistência a Saúde.

REICHENHEIM, M. E. \& MORAES, C. L., 2002. Buscando a qualidade das informações em pesquisas epidemiológicas. In: Caminhos do Pensamento: Epistemologia e Método (M. C. S. Minayo \& S. F. Deslandes, org.), pp. 227-254, Rio de Janeiro: Editora Fiocruz.

ROSSMAN, B. B. R.; HUGHES, H. M. \& ROSENBERG, M. S., 1999. Children and Interparental Violence: The Impact of Exposure. Philadelphia: Brunner/ Mazel. 
STRAUS, M. A., 1979. Measuring intra-familiar conflict and violence: The conflict tactics (CT) scales. Journal of Marriage and Family, 41:75-88.

STRAUS, M. A. \& GELLES, R. J., 1995. Physical violence in American families: Risk Factors and Adaptations to Violence in 8145 Families. New Brunswick: Transaction Publisher.

STRAUS, M. A. \& HAMBY, S. L., 1997. Measuring Physical and Psychological Maltreatment of Children with the Conflict Tactics Scales. In: Out of darkness: Contemporary Perspectives on Family Violence (G. K. Kantor \& J. L. Jasinski, ed.), pp. 119135, Thousand Oaks: Sage Publications Inc.

STRAUS, M. A.; HAMBY, S. L.; BONEY-MCCOY, S. \& SUGARM AN, D. B., 1996. The revised Conflict Tactics Scales (CTS2): Development and preliminary psychometric data. Journal of Family Issues, 17:283-316.
STRAUS, M. A.; HAMBY, S. H.; FINKELHOR, D.; MOORE, D. W. \& RUNYAN, D., 1998. Identification of child maltreatment with parent-child conflict tactics scales: Development and psychometric data for a national sample of American parents. Child Abuse \& Neglect, 22:249-270.

STREINER, D. L. \& NORMAN, G. R., 1995. Health Measurement Scales. A Practical Guide to their De velopment and Use. 2a Ed. Oxford: Oxford University Press.

WATTS, C. \& ZIM MERM AN, C., 2002. Violence against women: Global scope and magnitude. Lancet, 359:1232-1237.

WEISS, S.; GARZA, A.; CASALETTO, J.; STRATTON, M.; ERNST, A.; BLANTON, D. \& NICK, T. G., 2000. The out-of-hospital use of a domestic violence screen for assessing patient risk. Prehospital Emergency Care, 4:24-27.

Recebido em 6 de dezembro de 2002

Versão final reapresentada em 14 de abril de 2003

Aprovado em 25 de julho de 2003 